\title{
Naegleria fowleri induces MUC5AC and pro- inflammatory cytokines in human epithelial cells via ROS production and EGFR activation
}

\begin{abstract}
Correspondence
Mineko Shibayama

mineko@cinvestav.mx
\end{abstract}

Received 7 May 2009

Revised 9 July 2009

Accepted 30 July 2009

\author{
Isaac Cervantes-Sandoval, ${ }^{1,3}$ José de Jesús Serrano-Luna, ${ }^{2}$ \\ Patricia Meza-Cervantez, ${ }^{1}$ Rossana Arroyo, ${ }^{1}$ Víctor Tsutsumi ${ }^{1}$ \\ and Mineko Shibayama ${ }^{1}$
}

\begin{abstract}
${ }^{1}$ Department of Infectomic and Molecular Pathogenesis, Center for Research and Advanced Studies, National Polytechnic Institute, Av. IPN 2508, Mexico City 07360, Mexico

${ }^{2}$ Department of Cell Biology, Center for Research and Advanced Studies, National Polytechnic Institute, Av. IPN 2508, Mexico City 07360, Mexico

${ }^{3}$ Department of Immunology, National School of Biological Sciences, National Polytechnic Institute, Av. Manuel M. Carpio and Plan de Ayala, Mexico City 11340, Mexico
\end{abstract}

\begin{abstract}
Naegleria fowleri is an amoeboflagellate responsible for the fatal central nervous system (CNS) disease primary amoebic meningoencephalitis (PAM). This amoeba gains access to the CNS by invading the olfactory mucosa and crossing the cribriform plate. Studies using a mouse model of infection have shown that the host secretes mucus during the very early stages of infection, and this event is followed by an infiltration of neutrophils into the nasal cavity. In this study, we investigated the role of $N$. fowleri trophozoites in inducing the expression and secretion of airway mucin and pro-inflammatory mediators. Using the human mucoepidermal cell line $\mathrm{NCl}-\mathrm{H} 292$, we demonstrated that $N$. fowleri induced the expression of the MUC5AC gene and protein and the pro-inflammatory mediators interleukin-8 (IL-8) and interleukin-1 $\beta$ (IL-1 $\beta$ ), but not tumour necrosis factor- $\alpha$ or chemokine $\mathrm{c}-\mathrm{c}$ motif ligand 11 (eotaxin). Since the production of reactive oxygen species (ROS) is a common phenomenon involved in the signalling pathways of these molecules, we analysed if trophozoites were capable of causing ROS production in NCl-H292 cells by detecting oxidation of the fluorescent probe 2,7-dichlorofluorescein diacetate. $\mathrm{NCl}-\mathrm{H} 292$ cells generated ROS after 15-30 min of trophozoite stimulation. Furthermore, the expression of MUC5AC, IL-8 and IL-1 $\beta$ was inhibited in the presence of the ROS scavenger DMSO. In addition, the use of an epidermal growth factor receptor inhibitor decreased the expression of MUC5AC and IL-8, but not IL-1 $\beta$. We conclude that $N$. fowleri induces the expression of some host innate defence mechanisms, such as mucin secretion (MUC5AC) and local inflammation (IL-8 and IL-1 $\beta$ ) in respiratory epithelial cells via ROS production and suggest that these innate immune mechanisms probably prevent most PAM infections.
\end{abstract}

\section{INTRODUCTION}

Naegleria fowleri is an ubiquitous, free-living amoeba that has been isolated from diverse water and soil sources. Under certain conditions, which are not fully understood, these amoebae may infect the human central nervous

\footnotetext{
Abbreviations: CCL11, chemokine C-c motif ligand 11; CNS, central nervous system; EGFR, epidermal growth factor receptor; FDA, 2,7dichlorofluorescein diacetate; GAPDH, glyceraldehyde-3-phosphate dehydrogenase; IL-1 $\beta$, interleukin-1 $\beta$; IL-8, interleukin-8; MUC5AC, mucin 5AC; PAM, primary amoebic meningoencephalitis; PMA, phorbol 12-myristate 13-acetate; ROS, reactive oxygen species; TGF- $\alpha$, transforming growth factor- $\alpha$; TNF- $\alpha$, tumour necrosis factor- $\alpha$.
}

system (CNS), giving rise to primary amoebic meningoencephalitis (PAM). Infection usually occurs in young healthy people with a history of recent recreational water activities (Marciano-Cabral \& Cabral, 2007). Despite the ubiquity and worldwide distribution of these amoebae, only a little over 200 cases have been reported worldwide. Trophozoites invade the host by penetrating the olfactory neuroepithelium and migrating through the cribriform plate, before reaching the olfactory bulbs (Carter, 1970; John \& Nussbaum, 1983; Jarolim et al., 2000; Rojas-Hernández et al., 2004). Once in the CNS, the amoebae induce a strong inflammatory reaction, haemorrhage and lytic necrosis (Carter, 1970; Cervantes-Sandoval et al., 2008a). 
Immunohistochemical studies during the initial stages of infection have shown the presence of several trophozoites embedded within the mucus in the nasal cavity. This event is followed by the appearance of inflammatory cells, mainly neutrophils, surrounding the amoebae (Rojas-Hernández et al., 2004; Cervantes-Sandoval et al., 2008b). In order to improve our understanding of the initial stages of infection, it is necessary to study the molecular mechanisms involved in the activation of the inflammatory cells and other innate immune responses, and how these responses may either protect from or contribute to the pathogenesis of PAM.

In the present study, we evaluated the ability of $N$. fowleri trophozoites to induce the expression of the MUC5AC gene and protein, and the pro-inflammatory cytokines interleukin-8 (IL-8), interleukin-1 $\beta$ (IL-1 $\beta$ ), tumour necrosis factor- $\alpha$ (TNF- $\alpha)$ and the unrelated eosinophil chemokine $\mathrm{c}-\mathrm{c}$ motif ligand 11 (CCL11; eotaxin). In addition, we investigated some of the signalling routes involved in this activation, such as the generation of reactive oxygen species (ROS) and activation of epidermal growth factor receptor (EGFR). We used the intensively studied human airway epithelial cell line NCI-H292, which expresses the main human and mouse airway mucin MUC5AC and pro-inflammatory cytokines, in response to certain stimuli (Shao et al., 2003; Shao \& Nadel, 2005; Nakanaga et al., 2007). The results showed that N. fowleri trophozoites induced the expression of MUC5AC, IL-8 and IL- $1 \beta$, but not TNF- $\alpha$ or CCL11, in NCI-H292 cells. The expression was time-dependent, with peaks at 6 and $12 \mathrm{~h}$ following stimulation. $N$. fowleri trophozoites also induced the production of ROS after 30 and $60 \mathrm{~min}$. MUC5AC, IL8 and IL- $1 \beta$ expression were inhibited by the ROS scavenger DMSO, confirming the involvement of these mediators in the signalling cascade leading to the expression of these innate immune response products. Finally, the expression of MUC5AC and IL-8, but not IL$1 \beta$, was blocked by a specific inhibitor of EGFR, as previously reported, using phorbol 12-myristate 13-acetate (PMA) as a stimulus (Nakanaga et al., 2007). These results suggest that $N$. fowleri trophozoites induce innate immune responses in epithelial cells, such as NCI-H292, during the early stages of infection. Secretion of mucins and expression of pro-inflammatory cytokines are some of these responses, which are at least partially dependent on ROS generation and EGFR activation.

\section{METHODS}

Amoebic and cell cultures. The pathogenic strain N. fowleri ATCC 30808 was used in all experiments. Trophozoites were axenically cultured in $2 \%(\mathrm{w} / \mathrm{v})$ bactocasitone medium supplemented with $10 \%$ (v/v) fetal bovine serum (FBS; Equitech-bio) at $37^{\circ} \mathrm{C}$. Trophozoites were harvested during the exponential growth phase $(48 \mathrm{~h})$.

NCI-H292 cells, which have been widely used as a model for studies of mucin and innate response generation, were grown in RPMI 1640 medium with $10 \%$ (v/v) FBS (Gibco) and $25 \mathrm{mM}$ HEPES at $37^{\circ} \mathrm{C}$, in a $5 \% \mathrm{CO}_{2}$ atmosphere. After reaching semi-confluence $\left(80 \% ; 5 \times 10^{5}\right.$ cells per six-well culture plate), they were serum-starved for $24 \mathrm{~h}$ to maintain low basal levels of mucin expression. After $24 \mathrm{~h}$ of serum starvation, cells were treated with different stimuli.

Experimental animals. Experiments with animals were performed as previously described (Cervantes-Sandoval et al., 2008a). Briefly, 4week-old male $\mathrm{BALB} / \mathrm{c}$ mice were lightly anaesthetized in a chamber, using ether, and inoculated by the nasal route with $2.5 \times 10^{4}$ live $N$. fowleri trophozoites in $20 \mu \mathrm{l}$. After different post-inoculation intervals (1, 6 and $12 \mathrm{~h}$ ), the animals were euthanized and the brains were fixed. The skulls were decalcified and the brain tissue was processed and embedded in paraffin wax. Sections were cut at $7 \mu \mathrm{m}$ and stained using a rabbit polyclonal anti- $N$. fowleri antibody produced in our laboratory [diluted 1:50 in PBS-Tween $0.05 \%(\mathrm{v} / \mathrm{v})$ ] and a secondary antibody, anti-rabbit IgG labelled with peroxidase (diluted $1: 200$; Sigma-Aldrich). Colour was developed using a diaminobenzidine kit (Pierce).

The protocols for animal care were previously approved by the Institutional Committee (IACUC; ID number 244/05). The institution fulfils all the technical specifications for the production, care and use of laboratory animals and is certified by a National Law (NOM-062-ZOO-1999). All mice were handled according to the guidelines of the 2000 AVMA Panel of Euthanasia.

Expression of MUC5AC and pro-inflammatory cytokines. To determine if $N$. fowleri trophozoites were able to induce an innate immune response in the mucoepithelial NCI-H292 cells, serumstarved cells were co-incubated with $5 \times 10^{5}$ amoebae at a ratio of $1: 1$, for $1-6 \mathrm{~h}$. After $6 \mathrm{~h}$, amoebae were removed by cooling the medium for $5 \mathrm{~min}$, and the incubation continued up to 12 and $24 \mathrm{~h}$. This procedure was necessary because co-incubation with amoebae for longer than $6 \mathrm{~h}$ resulted in damage of the cell monolayer. $N$. fowleri-conditioned medium was prepared as described previously (Serrano-Luna et al., 2007) and was added to NCI-H292 cells for different time periods (1, 3, 6, 12 and $24 \mathrm{~h})$. For positive controls, cells were stimulated with PMA, $10 \mathrm{ng} \mathrm{ml}^{-1}$ for $1 \mathrm{~h}$, then washed three times with serum-free medium and cultured for an additional $24 \mathrm{~h}$. For negative controls, cells were incubated in RPMI medium without serum for $24 \mathrm{~h}$ (to maintain basal levels of expression). Finally, cell culture supernatants and cell lysates were collected to measure the expression of MUC5AC protein and cytokine mRNAs (IL-8, IL- $1 \beta$, TNF- $\alpha$ and CCL11).

MUC5AC protein determination. MUC5AC protein was determined by immunocytochemistry and dot-blots. For immunocytochemistry, stimulated and non-stimulated cells were fixed with $2 \%$ $(\mathrm{w} / \mathrm{v})$ paraformaldehyde and stained with mouse monoclonal antibody to MUC5AC (clone 1-13M1, Abcam) and our rabbit polyclonal anti-N. fowleri antibody (see above). The secondary antibodies used were goat anti-mouse IgG labelled with FITC (Zymed) and goat anti-rabbit IgG labelled with Cy5 (Zymed). Nuclei were stained with propidium iodide. Samples were examined under a confocal microscope (Olympus IX71).

NCI-H292 cell lysates were obtained using a lysis buffer [150 mM $\mathrm{NaCl} ; 1 \%$ (v/v) Triton X-100; $50 \mathrm{mM}$ Trizma]. For dot-blots, $2 \mu \mathrm{l}$ samples of total crude cell lysates (protein concentration adjusted to $5 \mu \mathrm{ml}^{-1}$ ) or conditioned cultured medium were absorbed onto a nitrocellulose membrane and blocked with $5 \%(\mathrm{w} / \mathrm{v})$ skimmed milk for $1 \mathrm{~h}$. Then the membrane was washed four times with PBS-Tween at $0.05 \%(\mathrm{v} / \mathrm{v})$ and incubated with the same monoclonal antibody to MUC5AC for $2 \mathrm{~h}$. The membrane was washed four times and incubated with a secondary antibody against mouse IgG labelled with horseradish peroxidase. Finally the membrane was washed six times and revealed with luminol kit reagent (Santa Cruz Biotechnology). As controls, fresh RPMI culture medium or lysis buffer were tested for 
the presence of MUC5AC; these tests gave negative results. The optical density of the dots was analysed using the NIH image software ImageJ (http://rsb.info.nih.gov/nih-image). Results are presented as one representative blot with the densitometry analysis of at least three replicates with similar results.

Analysis of MUC5AC and cytokine mRNA expression by RTPCR. In order to evaluate the levels of mucin and cytokine messenger expression after stimulation, total RNA was isolated using the QIAamp RNA blood mini kit (Qiagen), according to the manufacturer's procedure. mRNA expression was then analysed by RT-PCR using the One-Step RT-PCR kit (Qiagen), according to the manufacturer's instructions, and using the specific primers for MUC5AC, IL-8, IL-1 $\beta$, TNF- $\alpha$, CCL11 and glyceraldehyde-3-phosphate dehydrogenase (GAPDH) listed in Table 1. Primers without references were designed using the software Primer3 plus (http:// www.bioinformatics.nl/cgi-bin/primer3plus/primer3plus.cgi). PCR products were analysed by electrophoresis in $2 \%(\mathrm{w} / \mathrm{v})$ agarose gels, stained with ethidium bromide and visualized in a UV transilluminator (Bio-Rad). To measure the expression levels, bands in gels were analysed using the ImageJ. Results are presented as one representative gel with the densitometry analysis of at least three replicates with similar results. All PCR products were purified and sequenced to verify their identity. Briefly, the PCR products were polyadenylated, inserted in the pCR4-TOPO plasmid (Invitrogen), and used to transform Escherichia coli DH5 $\alpha$. Bacteria were seeded in solid LB medium containing ampicillin $\left(5 \mu \mathrm{g} \mathrm{ml}^{-1}\right)$ and grown for $24 \mathrm{~h}$. Further selection of positive colonies was made by PCR. Positive bacteria were again grown in liquid LB medium and the plasmid was purified and sequenced using the M13 sequencing primers. The results showed that the sequences were $100 \%$ homologous to the predicted sequences (data not shown).

ROS analysis. We determined the capacity of $N$. fowleri trophozoites to generate ROS in NCI-H292 cells. NCI-H292 cells were grown in six-well culture plates until confluence was reached. They were then maintained in RPMI culture medium without serum for $24 \mathrm{~h}$ prior to stimulation. The cells were stimulated with $5 \times 10^{5}$ trophozoites $(1: 1$ ratio, as mentioned above), or $10 \mathrm{ng} \mathrm{PMA} \mathrm{ml}^{-1}$ as a positive control, for 15,30 or 60 min in RPMI without serum and supplemented with $30 \mu \mathrm{M}$ 2,7-dichlorofluorescein diacetate (FDA). After the interaction time, the cell monolayers were washed four times with cold PBS to remove trophozoites and disaggregated using trypsin for $5 \mathrm{~min}$. Finally, they were washed three times with PBS and fluorescence was quantified in a fluorometer with an excitation wavelength of $485 \mathrm{~nm}$ and emission at $525 \mathrm{~nm}$. The fluorescence emitted by the nonstimulated cells was taken as the baseline level of ROS. The results are reported as mean $\pm S D$ of the increase over baseline value. The significance of the differences was evaluated using $t$-test, $P<0.05$.
Effect of DMSO and tyrphostin AG1478. We examined the roles of ROS and activation of the EGFR in the expression of proinflammatory cytokines and mucin. Cells were incubated with trophozoites for $6 \mathrm{~h}$, then they were removed from the culture medium and the incubation continued up to $24 \mathrm{~h}$, as mentioned above, in the presence of $1 \%(\mathrm{v} / \mathrm{v})$ DMSO, a compound that captures and inhibits the activity of ROS. In parallel assays, the cells were preincubated with $10 \mu \mathrm{M}$ tyrphostin AG1478, a strong inhibitor of EGFR, for $30 \mathrm{~min}$ before the stimulus with amoebae, as mentioned in the previous assay. After inhibition, cells were interacted with the stimuli and MUC5AC and cytokine expression were analysed as described above.

\section{RESULTS}

\section{N. fowleri induces mucus secretion and neutrophil infiltration in the mouse nasal cavity}

To corroborate previous observations of experimental PAM during the early stages of infection, we inoculated the nasal cavities of mice with live $N$. fowleri trophozoites and determined the induction of mucus secretion. The host mucosa reacted as early as $1 \mathrm{~h}$ post-inoculation by strong production and secretion of mucus, as previously reported (Rojas-Hernández et al., 2004; Cervantes-Sandoval et al., 2008b) (data not shown). Before contacting the epithelial cells, some trophozoites were intermixed with mucus in the lumen of the nasal cavity. Six hours post-inoculation, amoebae were seen covered by the mucus secretion and surrounded by a strong acute inflammatory reaction, composed mainly of neutrophils. Control animals inoculated with fresh culture medium showed no changes in the olfactory neuroepithelium (data not shown). These results suggest that the mouse epithelial cells responded to amoebic infection by exerting an innate immune response characterized by the production of mucus and proinflammatory signals. We used an in vitro approach to further elucidate the mechanisms involved in this response.

\section{$N$. fowleri induces the expression of MUC5AC in human mucoepithelial cells}

We evaluated the ability of $N$. fowleri trophozoites to induce the expression of mucins, particularly MUC5AC,

Table 1. RT-PCR primers

\begin{tabular}{|c|c|c|c|c|}
\hline $\begin{array}{l}\text { Protein } \\
\text { encoded }\end{array}$ & Primer A & Primer B & $\begin{array}{l}\text { Annealing } \\
\text { temp. }\left({ }^{\circ} \mathrm{C}\right)\end{array}$ & Reference \\
\hline MUC5AC & $5^{\prime}$-CAC CAA ATA CGC CAA CAA GAC-3' & $5^{\prime}$-CAG GGC CAC GCA GCC AGA GAA-3' & 61 & Shimizu et al. (2003) \\
\hline IL-8 & 5'-ATG ACT TCC AAG CTG GCC GTG-3' & 5'-CTC AGC CCT CTT CAA AAA CTT-3' & 58 & Teruya et al. (2007) \\
\hline TNF- $\alpha$ & 5'-TCC TTC AGA CAC CCT CAA CC-3' & 5'-AGG CCC CAG TTT GAA TTC TT-3' & 53 & Primer3 plus \\
\hline IL-1 $\beta$ & $5^{\prime}$-GGG CCT CAA GCA AAA GAA TC-3' & 5'-TTC TGC TTG AGA GGT GCT GA-3' & 55 & Primer3 plus \\
\hline CCL11 & $5^{\prime}$-AGA AAC CAC CAC CTC TCA CG-3' & 5'-CAC AGC TTT CTG GGG ACA TT-3' & 55 & Primer3 plus \\
\hline GAPDH & $\begin{array}{l}5^{\prime} \text {-CCA CCC ATG GCA AAT TCC ATG } \\
\text { GCA-3' }\end{array}$ & $\begin{array}{l}\text { 5'-TCT AGA CGG CAG GTC AGG TCC } \\
\text { ACC-3' }\end{array}$ & 66 & Shimizu et al. (2003) \\
\hline
\end{tabular}


which is the most abundant mucin in the airway epithelium in humans and mice, and has been reported to be overexpressed following diverse chemical and biological stimuli (Shao et al., 2003). We used NCI-H292 cells, which have been widely used to study upper-airway mucins, including MUC5AC, and other innate immune mediators. NCI-H292 cells were co-incubated with conditioned medium or $N$. fowleri trophozoites for different periods of time; the production of MUC5AC protein was determined by immunocytochemistry and dot-blots, and the expression of mRNA by RT-PCR. Confocal microscopy using a secondary antibody coupled to FITC showed that $N$. fowleri was able to strongly induce MUC5AC, compared with control, non-stimulated cells, which showed no MUC5AC-positive cells (Fig. 1a). MUC5AC production was apparent after $3 \mathrm{~h}$ interaction with trophozoites stained with a secondary antibody coupled to Cy5 (blue), and expression increased up to $24 \mathrm{~h}$ of co-incubation (Fig. 1a). The determination of MUC5AC protein by dotblots indicated that $N$. fowleri was able to induce MUC5AC (a)
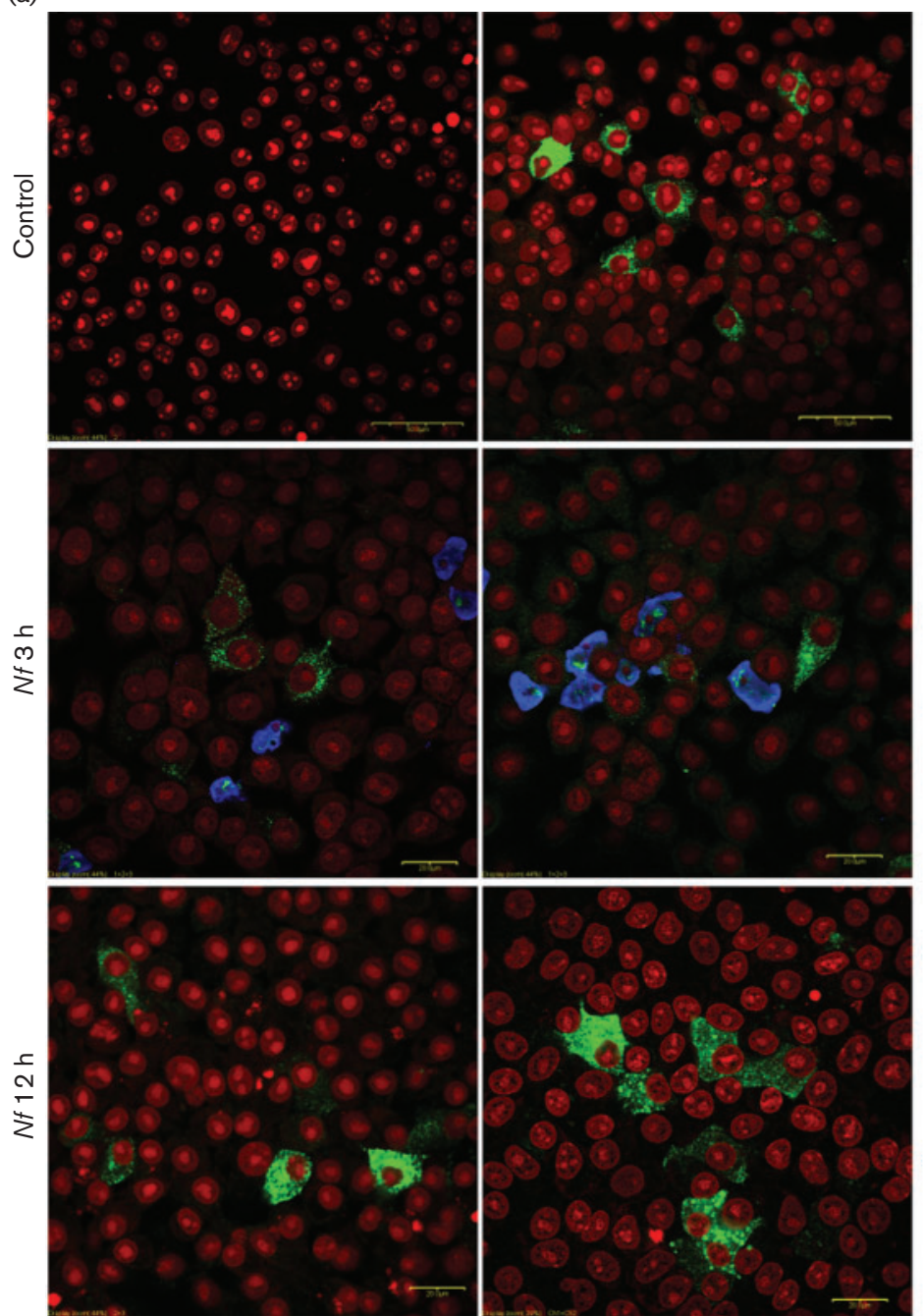

(b)

\begin{tabular}{|c|c|c|c|c|c|c|c|c|}
\hline \multirow{3}{*}{ Time (h) } & \multicolumn{5}{|c|}{ N. fowleri } & \multirow[b]{2}{*}{$\mathrm{FM} \mathrm{Nf}$} & \multirow[b]{2}{*}{ SS } & \\
\hline & 1 & 3 & 6 & 12 & 24 & & & PMA \\
\hline & 0 & 0 & 0 & 0 & 0 & & 3 & \\
\hline
\end{tabular}

$\sum_{0}^{\nwarrow}$

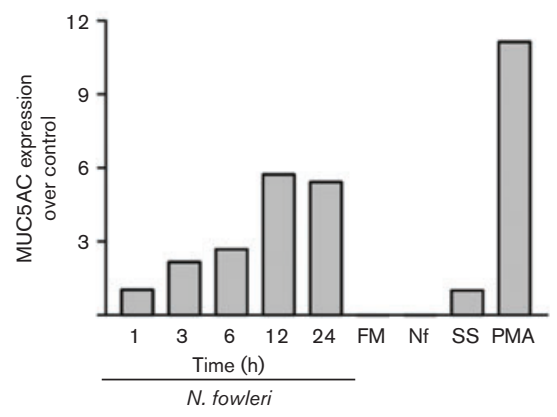

(c)
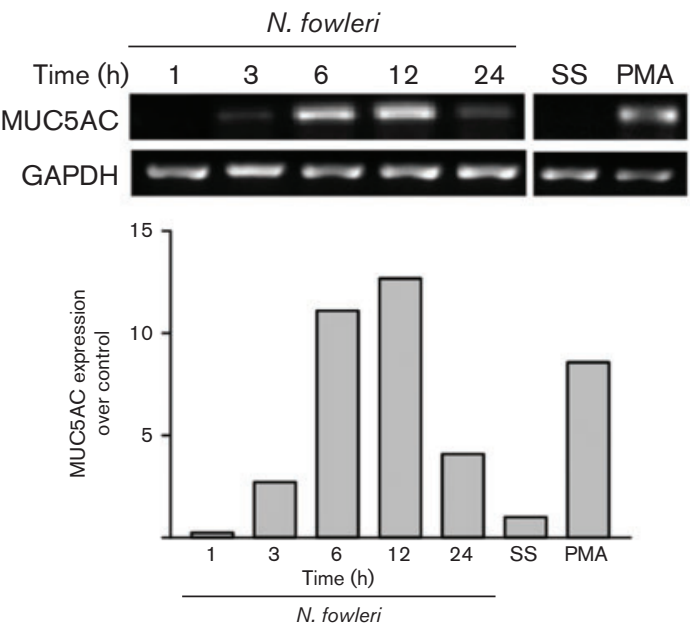

Fig. 1. MUC5AC expression in $\mathrm{NCl}-\mathrm{H} 292$ epithelial cells induced by $\mathrm{N}$. fowleri. (a) Confocal microscopy shows the production of MUC5AC (FITC) by NCl-H292 cells stimulated by trophozoites (Cy5, Nf) or PMA, and by non-stimulated cells (control), after $3,6,12$ and $24 \mathrm{~h}$. Cells were incubated with trophozoites for $6 \mathrm{~h}$, then they were removed from the culture medium and the incubation was continued up to 12 and 24 h. Nuclei were stained with propidium iodide. Scale bars, $20 \mu \mathrm{m}$. (b) MUC5AC protein expression determined by dot-blots after stimulation with $N$. fowleri trophozoites. Negative controls were serumcomplemented fresh medium (FM) and $N$. fowleri crude extract $(N f)$; control cells without stimulus were incubated in medium without serum (SS) and cells stimulated with PMA were used as positive expression control. Densitometric analysis results are shown below the blots. (c) Expression of MUC5AC RNA determined by RT-PCR after stimulation with N. fowleri trophozoites for different time points. Control cells without stimulus were incubated in medium without serum (SS) and cells stimulated with PMA were used as positive expression control. Densitometric analysis results are shown below the blots. 
expression in a time-dependent manner, reaching a maximum expression level of almost sixfold that of the control by $12 \mathrm{~h}$ after stimulation, which was maintained at $24 \mathrm{~h}$ (Fig. 1b). In the positive (PMA) control, the dot-blot showed an overproduction of almost 12-fold, in accordance with the confocal analysis. The non-stimulated, negative control (SS) showed very low basal production of MUC5AC protein (Fig. 1b). Similarly, we tested the fresh medium and the total amoebic crude extract for the presence of MUC5AC protein, giving negative results (Fig. 1b). Analysis of mRNA expression showed similar results. N. fowleri trophozoites induced the expression of MUC5AC mRNA in a time-dependent manner. Expression became apparent from $3 \mathrm{~h}$ and increased to reach a maximum of approximately 12-fold after 6 and $12 \mathrm{~h}$ (Fig. 1c). In contrast to protein analysis, the positive control showed a marked increase in expression (ninefold), but it was lower than the level induced by $N$. fowleri. This could be related to the different mechanisms of control and regulation of expression, for example the stability of mRNA. Nevertheless, further analysis is required to clarify these observations. As an amplification control, the reactions were adjusted to GAPDH mRNA expression (Fig. 1c). These results suggest that mucin secretion in NCI-H292 cells is an active process induced by the presence of $N$. fowleri.

\section{$N$. fowleri induces the expression of pro-inflammatory signals in human mucoepithelial cells}

Because the animal model shows an inflammatory infiltrate with secretion of mucus in the nasal cavity in response to
$N$. fowleri, we evaluated the effect of trophozoites on the induction of mRNA for some pro-inflammatory cytokines (IL-8, TNF- $\alpha$, IL-1 $\beta$, CCL11) in NCI-H292 cells. N. fowleri trophozoites were co-incubated with NCI-H292 cells for different time periods and the mRNA expression of the different cytokines was determined. The amoebae induced the expression of IL- 8 from $3 \mathrm{~h}$ (threefold), as for MUC5AC, and expression continued to increase to a maximum level of 16-fold after $24 \mathrm{~h}$ of interaction (Fig. 2a, b), which was very similar to the expression pattern observed in the PMA-treated, positive control cells. Similarly, N. fowleri induced the expression of IL- $1 \beta$ after $3 \mathrm{~h}$ of interaction (fivefold), and levels increased to almost 13 -fold after $24 \mathrm{~h}$. The highest level of IL-1 $\beta$ obtained after $N$. fowleri stimulation was lower than the levels obtained with PMA, which resulted in an increase of about 16-fold over the control. We observed basal expression levels of the pro-inflammatory cytokine TNF- $\alpha$ under all conditions studied, and this expression was not modified by stimulation with $N$. fowleri trophozoites (Fig. 2a, b). In contrast, PMA was able to induce a strong expression of TNF- $\alpha$. Finally, we evaluated expression of the unrelated pro-inflammatory cytokine CCL11, which is a strong chemotactic stimulator of eosinophils, which are not observed in vivo during the early stages of infection. As expected, neither N. fowleri trophozoites nor PMA were able to increase the production of CCL11 (Fig. 2a); we did not test alternative stimuli to demonstrate expression of CCL11 in these cells. As a positive control, we used genomic DNA to amplify CCL11 under the same RT-PCR conditions, and obtained a product of the predicted size (Fig. 2a). These results indicate that $N$. fowleri induced the expression of the pro-inflammatory cytokines IL-1 $\beta$ and (a)

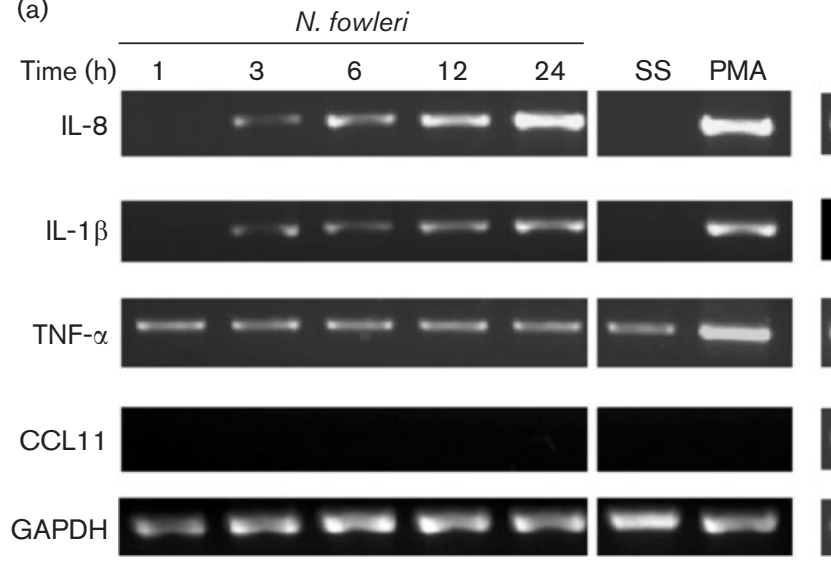

(b)
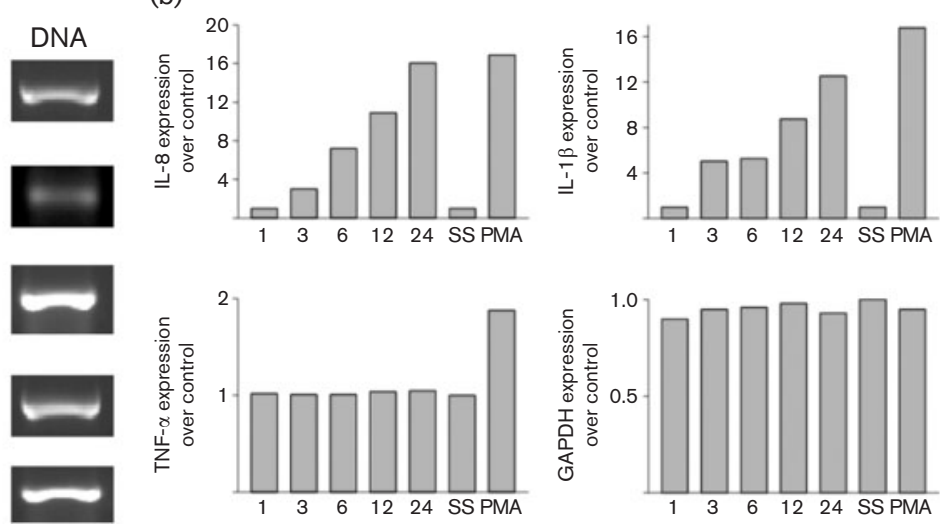

Fig. 2. $N$. fowleri induces the expression of pro-inflammatory cytokines in NCl-H292 epithelial cells. (a) Determination of expression levels of IL-8, IL-1 $\beta$, TNF- $\alpha$, CCL11 and GAPDH (housekeeping) mRNAs by RT-PCR, after different periods of stimulation with $N$. fowleri trophozoites. Cells were incubated with trophozoites for $6 \mathrm{~h}$, then they were removed from the culture medium and the incubation was continued up to $24 \mathrm{~h}$. Negative control cells were cultured in medium without serum (SS) and positive control cells were incubated with $10 \mathrm{ng} \mathrm{PMA} \mathrm{ml}^{-1}$. PCR of genomic DNA was used to validate the RT-PCR conditions. (b) Densitometric analysis of the bands obtained in (a). Results represent the expression levels of the different cytokines relative to the control. 
IL-8 in epithelial cells, and correlate with the infiltration of neutrophils into the nasal cavity observed in the animal model, since these are strong neutrophil-chemotactic molecules. To validate the amplified products, PCR was performed using genomic DNA to ensure that the conditions of the amplification reaction were appropriate; these controls were always positive (Fig. 2a). Similarly, all amplified fragments were purified and sequenced to verify their identities. The sequencing of amplicons showed $100 \%$ homology with sequences reported for the different mRNAs.

\section{N. fowleri increases production of ROS}

Because ROS have been reported to play a key role in regulating the expression of MUC5AC, IL- $1 \beta$ and IL- 8 , we determined if $N$. fowleri induced an increase in ROS and if this increase was involved in regulating the synthesis of these molecules. N. fowleri trophozoites were co-incubated with NCI-H292 cells for 15, 30 or $60 \mathrm{~min}$ in the presence of the ROS fluorescent probe FDA. ROS were slightly increased 15 min after addition of $N$. fowleri, but marked increases of up to 1.6 times that of the non-stimulated control cells occurred after $30 \mathrm{~min}$ of interaction, and up to 2.3 times after 60 min (Fig. 3). Positive control cells stimulated with PMA showed increases of up to 1.6, 1.9 and 3.4 times after 15, 30 and $60 \mathrm{~min}$ of interaction, respectively (Fig. 3). These results strongly suggest that an increase in ROS may be involved in the positive regulation of cytokines and mucin MUC5AC.

\section{ROS production and EGFR activation are required for MUC5AC and cytokine overexpression}

As mentioned previously, ROS generation has been reported to be important for the upregulation of several

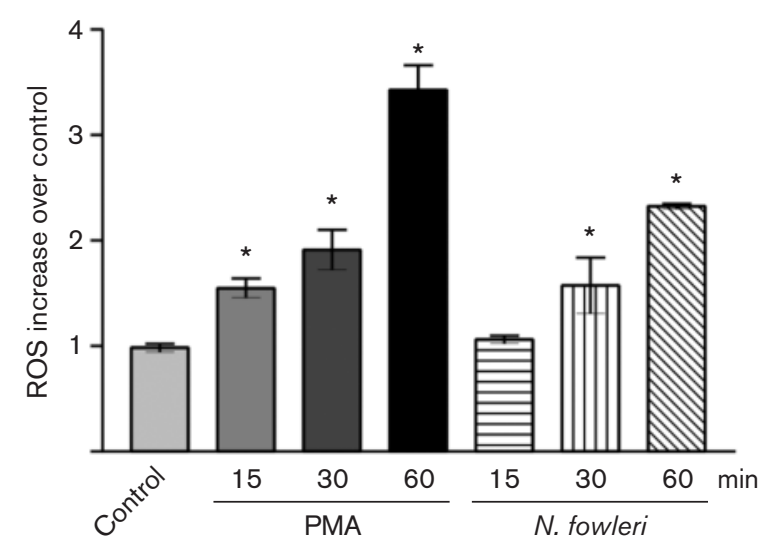

Fig. 3. $N$. fowleri induces the production of ROS. ROS were determined by FDA fluorescence in $\mathrm{NCl}-\mathrm{H} 292$ cells stimulated with PMA as positive control, or with $N$. fowleri trophozoites, for different time periods $(15,30$ and $60 \mathrm{~min})$. Data show the production of ROS relative to that in control, non-stimulated cells. Values represent the means \pm SD of three replicate experiments; ${ }^{\star} P<0.05 \%, t$-test. molecules involved in innate immune responses (Hsu \& Wen, 2002). The above results suggest that the increase in ROS observed a few minutes after $N$. fowleri stimulation may be involved in the upregulation of MUC5AC, IL- 8 and IL- $1 \beta$. To test this hypothesis, we stimulated NCI-H292 cells with trophozoites for $6 \mathrm{~h}$, then the amoebae were removed and the incubation was continued for $24 \mathrm{~h}$ in the presence of $1 \%$ DMSO (a ROS scavenger). After the interaction, we measured the mRNA expression of the molecules previously tested. Inhibition of the activity of ROS with DMSO reduced the expression levels of MUC5AC, IL-8, IL- $1 \beta$ to $0 \%, 21 \%$ and $32 \%$, respectively, when expression levels in the absence of DMSO were taken as $100 \%$ (Fig. 4a, b). Similar results were obtained in cells interacted with PMA as a positive control (data not shown). These results indicate that $N$. fowleri is able to increase the levels of ROS, and that this increase is significant and is probably involved in the signalling pathway leading to increased expression of MUC5AC, IL-8 and IL- $1 \beta$.

It has also been shown that ROS are involved in the signalling pathway leading to IL-8 and MUC5AC production, by activation of EGFR. To demonstrate if activation of this receptor is involved in the expression of MUC5AC, IL- $1 \beta$ and IL- 8 induced by $N$. fowleri, NCI-H292 cells were stimulated with trophozoites as in the previous assay, after pre-incubation for 30 min with $10 \mu \mathrm{M}$ tyrphostin AG1478, a specific inhibitor of EGFR phosphorylation (activation). Total RNA was extracted from the cells and the levels of MUC5AC, IL- 8 and IL- $1 \beta$ expression were analysed by RTPCR. MUC5AC and IL-8 expression levels were reduced after specific inhibition of EGFR. MUC5AC levels were reduced to $0 \%$, and IL-8 levels to $41 \%$ (Fig. $4 \mathrm{a}, \mathrm{b}$ ). Again, similar results were obtained in cells interacted with PMA as a positive control (data not shown). These results indicate that EGFR activation is necessary to induce the production of these proteins. In contrast, the expression of IL- $1 \beta$ was not inhibited by tyrphostin AG1478, indicating that EGFR is not involved in regulating the expression of this cytokine (Fig. 4a, b). When we combined both inhibitors (DMSO and tyrphostin), the results were similar to those obtained with only DMSO: low expression of IL-8 and IL- $1 \beta$ was still observed (Fig. $4 \mathrm{a}, \mathrm{b}$ ). This result indicates that the effect of these inhibitors is not synergistic, and perhaps another kind of receptor or signalling pathway participates in the production of these pro-inflammatory cytokines.

\section{DISCUSSION}

$N$. fowleri is a free-living amoeboflagellate that can infect humans by invading the nasal tissues. The infection usually occurs after performing aquatic activities in water bodies containing the parasite (Schuster \& Visvesvara, 2004; Marciano-Cabral \& Cabral, 2007). Histopathological studies using a mouse model showed that the amoebae infect the host by penetrating the olfactory mucosa, 
(a)

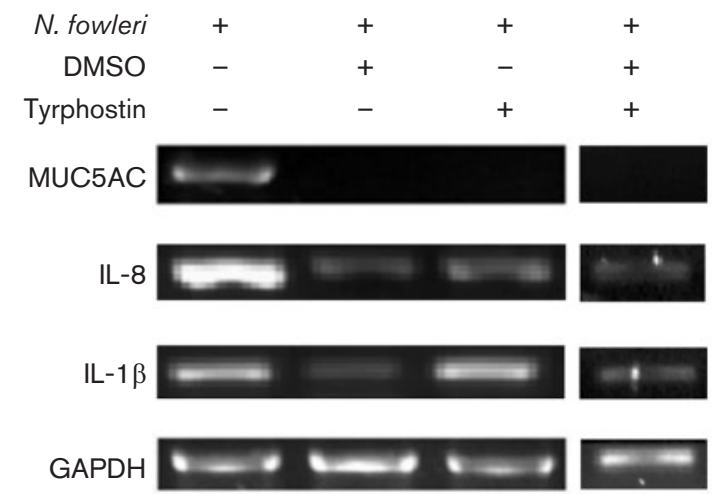

(b)

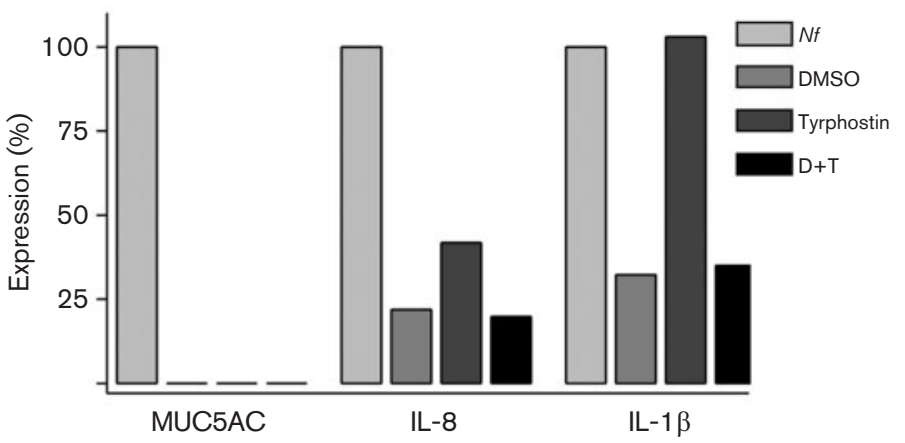

Fig. 4. Role of ROS and EGFR in MUC5AC and cytokine expression. (a) RT-PCR determination of the effect of DMSO (ROS scavenger), tyrphostin (EGFR inhibitor) or both ( $D+T$ ) on the expression of MUC5AC and pro-inflammatory cytokines in NClH292 cells after interaction with N. fowleri trophozoites. (b) Densitometric analysis of the gels in (a), showing the percentage expression of the different mRNAs, relative to the expression levels observed in cells stimulated without the presence of any inhibitor (100\%).

migrating through the olfactory nerves, and passing through the cribriform plate to reach the olfactory bulbs of the CNS, where an intense inflammatory reaction is produced (Carter, 1970; Jarolim et al., 2000, 2002; Cervantes-Sandoval et al., 2008a). In the very early stages of infection, trophozoites are seen in the lumen of the nasal cavity embedded in a large amount of mucus and surrounded by infiltrated neutrophils (Thong et al., 1983; Rojas-Hernández et al., 2004; Cervantes-Sandoval et al., $2008 \mathrm{~b})$. At this stage of infection, mucin secretion, inflammation and other local innate responses could be important protective barriers against infection. It has been reported that the presence of humoral (Edwards et al., 1976) and cellular immunity (Cursons et al., 1980) may be protective against Naegleria infections. This protection is probably mediated by primed neutrophils killing trophozoites, in which antibodies play an important role in the opsonization of the parasites (Ferrante \& Mocatta, 1984; Ferrante, 1989); the alternative activation pathway of the complement could also be involved (Rowan-Kelly et al., 1980).

In this study, we determined if the processes of mucin hypersecretion and inflammation observed in the animal model were active processes stimulated directly by the presence of the parasite. We used a widely studied in vitro model comprising mucin production by NCI-H292 cells, an immortal cell line derived from a human cancer, which responds to a variety of chemical and biological stimuli such as $\mathrm{H}_{2} \mathrm{O}_{2}$ (Takeyama et al., 2000) and LPS (Smirnova et al., 2003). Although olfactory epithelium does not usually contain mucin-producing goblet cells, this epithelium is a relatively small and limited region at the roof of the nasal cavity and behind the nostrils, and is closely associated with an epithelium of the upper respiratory type that contains abundant goblet cells. So mucin secretion by these cells could be important, since trophozoites must pass the respiratory epithelium in the nasal cavity before approaching and invading the olfactory epithelium.

We showed that $N$. fowleri was capable of inducing the expression and secretion of MUC5AC in vitro. In a previous report, we demonstrated that MUC5AC and other mucins could be important resistance factors protecting against $N$. fowleri infection by inhibiting adhesion (Cervantes-Sandoval et al., 2008b). MUC5AC is the most abundant mucin in the upper respiratory tract and is secreted mainly by goblet cells or ciliated epithelial cells differentiated to mucin-producing cells (Rose et al., 2001; Kim et al., 2005). It polymerizes to form a thick gel with protective properties. Recent studies have suggested that ROS play a key role in the signalling pathways involved in inducing the expression of MUC5AC (Takeyama et al., 2000). Stimulation of NCI-H292 cells with $\mathrm{H}_{2} \mathrm{O}_{2}$ activates the production of ROS, leading to the expression of MUC5AC (Takeyama et al., 2000) via activation of the EGFR (Takeyama et al., 1999). In order to determine if this signalling pathway is involved in the expression of MUC5AC induced by $N$. fowleri, we measured ROS in cells stimulated with this amoeba. The results showed that $N$. fowleri increased the production of ROS in the epithelial cell line NCI-H292, starting after a few minutes of interaction. This result suggests that ROS could be involved in the activation of the cellular response by the external agent (amoebae), and could be important in activating various signalling pathways, including those leading to the production of MUC5AC. To prove this hypothesis, we determined the importance of ROS in the induction of MUC5AC, by treating NCI-H292 cells with the ROS scavenger DMSO. During stimulation with amoebae, DMSO significantly decreased the expression of MUC5AC induced by N. fowleri or PMA, so confirming the 
involvement of ROS in regulating mucin expression. The most important outcome of the increase in ROS during the regulation of mucin expression is the activation of EGFR (Takeyama et al., 2000). Pre-incubation of cells with DMSO for $30 \mathrm{~min}$ prior to stimulation with amoebae abolished the production of MUC5AC. These results confirmed that stimulation with $N$. fowleri leads to an increase in ROS, which in turn leads to activation of the EGFR, which is involved in the regulation of MUC5AC. Similarly, N. fowleri was found to induce the expression of the pro-inflammatory cytokines IL- $1 \beta$ and IL-8. This induction is in accordance with the strong inflammatory infiltrate, composed mainly of neutrophils, present in the nasal cavity prior to the invasion of tissues. IL-8 is one of the strongest neutrophil chemoattractants (Ibelgaufts, 1995) and specifically activates these inflammatory cells. Here, we showed that both tyrphostin and DMSO significantly inhibited the expression of IL-8 in NCIH292 cells, indicating that $N$. fowleri stimulates the production of IL- 8 and MUC5AC by a shared mechanism as reported by Nakanaga et al. (2007).

On the other hand, IL- $1 \beta$ is a molecule with various functions and is an important mediator of inflammatory reactions. The in vivo injection of this cytokine induces the local accumulation of neutrophils at the site of inoculation, as well as the activation of oxidative metabolism of neutrophils (Ibelgaufts, 1995). Similar to the situation for MUC5AC and IL-8, ROS have been shown to be involved in the regulation of IL-1 $\beta$ (Hsu \& Wen, 2002). Moreover, it has been determined that IL-1 is positively regulated through different signalling pathways, which may depend on p38, JNK or MEK, according to the stimulus, but that all the pathways are dependent on an increase in ROS (Kim et al., 2004). In the present study, we showed that inhibition of ROS by DMSO also reduced the expression of IL- $1 \beta$ after stimulation by the amoebae. However, unlike the situation with MUC5AC and IL-8, EGFR inhibition did not affect the expression of IL- $1 \beta$, suggesting that these molecules do not share the same signalling pathway, except for their dependence on ROS generation. Furthermore, it was noted that $N$. fowleri was incapable of inducing the expression of CCL11, as expected, since CCL11 is an eosinophil chemoattractant, and no eosinophils are observed in the nasal cavity in vivo. In contrast to positive control cells stimulated with PMA, N. fowleri trophozoites were incapable of inducing TNF- $\alpha$. Production of this molecule is increased in acute events triggered by different stimuli, and it has been shown to be involved in the production of mucin in the nasal epithelium. PMA must induce signalling pathways that are different from those produced by $N$. fowleri. Nevertheless, these results need to be confirmed using different epithelial cell lines or primary culture cells, since immortal cell cultures are not always representative of the in vivo function.

In conclusion, we have determined that $N$. fowleri is capable of activating epithelial cells and that this activation leads to increased ROS production, which in turn helps to increase the expression of MUC5AC and IL-8 by an EGFR-dependent mechanism and of IL- $1 \beta$ by an EGFRindependent mechanism. These results are consistent with observations at early stages of infection in the in vivo model, characterized by the presence of parasites in the nasal cavity surrounded by abundant mucus and inflammatory infiltrates rich in neutrophils. We propose that these initial natural immune responses, together with specific cellular and humoral immunity (Cursons et al., 1980), including activated neutrophils (Ferrante \& Mocatta, 1984; Ferrante, 1989; Ferrante et al., 1988, 1989 ) and the alternative activation of complement (Rowan-Kelly et al., 1980) may all participate in the prevention of most $N$. fowleri infections. More studies are needed to further increase our understanding of the early events occurring during PAM pathogenesis and the means by which the host generates an effective innate response against $N$. fowleri invasion.

\section{ACKNOWLEDGEMENTS}

The authors acknowledge Angélica Silva Olivares and Silvia Galindo Gómez for their invaluable technical support. The authors also thank Juana Narváez for confocal assistance. This work was supported by CONACyT grant 191769.

\section{REFERENCES}

Carter, R. F. (1970). Description of a Naegleria sp. isolated from two cases of primary amoebic meningo-encephalitis, and of the experimental pathological changes induced by it. J Pathol 100, 217-244.

Cervantes-Sandoval, I., Serrano-Luna, J. J., Garcia-Latorre, E., Tsutsumi, V. \& Shibayama, M. (2008a). Characterization of brain inflammation during primary amoebic meningoencephalitis. Parasitol Int 57, 307-313.

Cervantes-Sandoval, I., Serrano Luna, J. J., Garcia-Latorre, E., Tsutsumi, V. \& Shibayama, M. (2008b). Mucins in host defence against Naegleria fowleri and mucinolytic activity as a possible means of evasion. Microbiology 154, 3895-3904.

Cursons, R. T., Brown, T. J., Keys, E. A., Moriarty, K. M. \& Till, D. (1980). Immunity to pathogenic free-living amoebae: role of cellmediated immunity. Infect Immun 29, 408-410.

Edwards, J. H., Griffiths, A. J. \& Mullins, J. (1976). Protozoa as sources of antigen in 'humidifier fever'. Nature 264, 438-439.

Ferrante, A. (1989). Augmentation of the neutrophil response to the Naegleria fowleri by tumor necrosis factor alpha. Infect Immun 57, 3110-3115.

Ferrante, A. \& Mocatta, T. J. (1984). Human neutrophils require activation by mononuclear leucocytes conditioned medium to kill the pathogenic free-living amoeba, Naegleria fowleri. Clin Exp Immunol 56, 559-566.

Ferrante, A., Carter, R. F., Lopez, A. F., Rowan-Kelly, B., Hill, N. L. \& Vadas, M. A. (1988). Depression of immunity to Naegleria fowleri in mice by selective depletion of neutrophils with a monoclonal antibody. Infect Immun 56, 2286-2291.

Ferrante, A., Hill, N. L., Goh, D. H. \& Kumaritilake, L. (1989). Altered neutrophils in mice immune to experimental Naegleria amoebic meningoencephalitis. Immunol Lett 22, 301-305. 
Hsu, H. Y. \& Wen, M. H. (2002). Lipopolysaccharide-mediated reactive oxygen species and signal transduction in the regulation of interleukin-1 gene expression. J Biol Chem 277, 22131-22139.

Ibelgaufts, H. (1995). Cytokines Dictionary. Wenheim, Germany: Wiley-VCH.

Jarolim, K. L., McCosh, J. K., Howard, M. J. \& John, D. T. (2000). A light microscopy study of the migration of $N$. fowleri from the nasal submucosa to the central nervous system during the early stage of primary amebic meningoencephalitis in mice. J Parasitol 86, 50-55.

Jarolim, K. L., McCosh, J. K. \& Howard, M. J. (2002). The role of blood vessels and lungs in the dissemination of Naegleria fowleri following intranasal inoculation in mice. Folia Parasitol (Praha) 49, 183-188.

John, D. T. \& Nussbaum, S. L. (1983). Naegleria fowleri infection acquired by mice through swimming in amebae-contaminated water. J Parasitol 69, 871-874.

Kim, S. H., Smith, C. J. \& Van Eldik, L. J. (2004). Importance of MAPK pathways for microglial proinflammatory cytokine IL- $1 \beta$ production. Neurobiol Aging 25, 431-439.

Kim, S., Schein, A. J. \& Nadel, J. A. (2005). E-cadherin promotes EGFR-mediated cell differentiation and MUC5AC mucin expression in cultured human airway epithelial cells. Am J Physiol Lung Cell Mol Physiol 289, L1049-L1060.

Marciano-Cabral, F. \& Cabral, G. A. (2007). The immune response to Naegleria fowleri amoeba and pathogenesis of infection. FEMS Immunol Med Microbiol 51, 243-259.

Nakanaga, T., Nadel, J. A., Ueki, I. F., Koff, J. L. \& Shao, M. X. (2007). Regulation of interleukin 8 via an airway epithelial signaling cascade. Am J Physiol Lung Cell Mol Physiol 292, L1289-L1296.

Rojas-Hernández, S., Jarillo-Luna, A., Rodríguez-Monroy, M., Moreno-Fierros, L. \& Campos-Rodríguez, R. (2004). Immunohistochemical characterization of the initial stages of Naegleria fowleri meningoencephalitis in mice. Parasitol Res 94, 31-36.

Rose, M. C., Nickola, T. J. \& Voynow, J. A. (2001). Airway mucus obstruction: mucin glycoproteins, $M U C$ gene regulation and goblet cell hyperplasia. Am J Respir Cell Mol Biol 25, 533-537.

Rowan-Kelly, B., Ferrante, A. \& Thong, Y. H. (1980). Activation of complement by Naegleria. Trans $R$ Soc Trop Med Hyg 74, 333-336.
Schuster, F. L. \& Visvesvara, G. S. (2004). Free-living amoeba as opportunistic and non-opportunistic pathogens of humans and animals. Int J Parasitol 34, 1001-1027.

Serrano-Luna, J., Cervantes-Sandoval, I., Tsutsumi, V. \& Shibayama, M. (2007). Biochemical comparison of proteases from pathogenic Naegleria fowleri and non-pathogenic Naegleria gruberi. J Eukaryot Microbiol 54, 411-417.

Shao, M. X. \& Nadel, J. A. (2005). Neutrophil elastase induces MUC5AC mucin production in human airway epithelial cells via a cascade involving protein kinase $\mathrm{C}$, reactive oxygen species, and TNF$\alpha$-converting enzyme. J Immunol 175, 4009-4016.

Shao, M. X., Ueki, I. F. \& Nadel, J. A. (2003). Tumor necrosis factor $\alpha$ converting enzyme mediates MUC5AC mucin expression in cultured human airway epithelial cells. Proc Natl Acad Sci U S A 100, 1161811623 .

Shimizu, T., Shimizu, S., Hattori, R. \& Majima, Y. (2003). A mechanism of antigen-induced goblet cell degranulation in the nasal epithelium of sensitized rats. J Allergy Clin Immunol 112, 119-125.

Smirnova, M. G., Guo, L., Birchall, J. P. \& Pearson, J. P. (2003). LPS upregulates mucin and cytokine mRNA expression and stimulates mucin and cytokine secretion in goblet cells. Cell Immunol 221, 42-49.

Takeyama, K., Dabbagh, K., Lee, H. M., Agustí, C., Lausier, J. A., Ueki, I. F., Grattan, K. M. \& Nadel, J. A. (1999). Epidermal growth factor system regulates mucin production in airways. Proc Natl Acad Sci U S A 96, 3081-3086.

Takeyama, K., Dabbagh, K., Shim, J. J., Dao-Pick, T., Ueki, I. F. \& Nadel, J. A. (2000). Oxidative stress causes mucin synthesis via transcription of epidermal growth factor receptor: role of neutrophils. J Immunol 164, 1546-1552.

Teruya, H., Higa, F., Akamine, M., Ishikawa, C., Okudaira, T., Tomimori, K., Mukaida, N., Tateyama, M., Heuner, K. \& other authors (2007). Mechanisms of Legionella pneumophila-induced interleukin-8 expression in human lung epithelial cells. BMC Microbiol 7, 102.

Thong, Y. H., Carter, R. F., Ferrante, A. \& Kowan-Kelly, B. (1983). Site of expression of immunity to Naegleria fowleri in immunized mice. Parasite Immunol 5, 67-76.

Edited by: L. Knoll 